\title{
Knowledge and Practices Regarding Psychosocial Aspects of Palliative Care among Healthcare Workers in Ohangwena Region, Namibia
}

\author{
Article by Hileni Niikondo ${ }^{1}$, Kabwebwe H. Mitonga ${ }^{2}$ \\ ${ }^{1}$ MPH, Public Health, Texila American University \\ ${ }^{2}$ PHD, Public Health, University of Namibia \\ E-mail: hniikondo@unam.na
}

\begin{abstract}
In Namibia, palliative care service is necessitated by the situation of progressive life limiting illnesses, which demand access to psychosocially appropriate holistic palliative care. Little is known about the knowledge and practice of healthcare workers on the psychosocial aspects of holistic palliative care.

This study was conducted to critically analyze the knowledge and practice of healthcare workers on psychosocial aspects of holistic palliative care and identify their training needs.

A cross-sectional analytical study was conducted in the public health facilities in Ohangwena region, amongst the randomly selected sample of 56 healthcare workers. Structured questionnaires were self administered to capture the respondent's knowledge and skills.

Respondents mean age was $36.2 \pm 9.81$.Male respondents were 20 (35.71\%) while female were 36 (64.29\%). Of the 56 healthcare workers who participated in the study, 2 (3.6\%) were medical practitioners, 44 (78.6\%) nurses, 1 (1.8\%) physiotherapists, 3 (5.4\%) social workers and 6 (10.7\%)

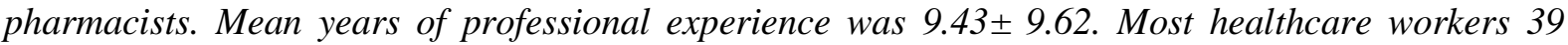
(69.6\%) indicated not received any training on holistic palliative care.

Knowledge of correct classification for morphine was associated with access to internet $(p<0.05)$. There was a significant association between those confronted by palliative care situation and knowledge of morphine $(p<0.05)$. Knowledge of pain intensity among children was significantly associated with access to internet $(p=0.01)$. Healthcare workers lack knowledge in the psychosocial aspects of holistic palliative care which can implicate the quality of care to patients. This study suggested training of healthcare workers as well as internet access.
\end{abstract}

Keywords: Palliative care, healthcare workers, knowledge, psychosocial care, pain, training

\section{Introduction}

Palliative care and psychosocial palliative care in particular is necessitated by the increased prevalence of life limiting conditions. Namibia has high rate of HIV/AIDS infection of 13.3\% among adults, that is contributing to the high demand of appropriate palliative care, which is part of human rights to healthcare (HPV Information Centre, 2014).In the same vein, Namibia has prevalence of cancer of different body systems which necessitate the need for palliative care. These include cancer of the cervix, cancer of the rectum, cancer of prostate gland and other organs. Patients with cancer endure many difficulties which include experiences of pain and psychological symptoms such as depression, social isolation and spiritual distress especially in the advanced stages of the disease (African Palliative Care Association, 2013). In addition, African Palliative Care Association (2013) also indicated that, family members of those afflicted by cancer also suffer emotional and financial burden related to the demand for care.

As the prevalence of life limiting illnesses increases, the clients' ability to self care diminishes, and more demand is put on healthcare workers as well as on the caregivers.

Therefore, training of healthcare workers in palliative care is necessary for them to have the appropriate knowledge, skills and competencies and to translate these experiences into practices. 
Moreover, training is required for the purpose of changing attitudes, beliefs and values, thus making the palliative care healthcare workers able to perform their duties effectively and ethically (APC, 2014).

Due to the increased need to provide palliative care services, the Ministry of Health and Social Services (MOHSS) of Namibia, complied with the demand of international request by drafting the palliative care policy. The policy indicated that healthcare workers who provide palliative care should be trained to obtain the knowledge and competence into the area of palliative care as per guidelines (Ministry of Health and Social Services, 2013), (African Palliative Care Association, 2011). Equally of important is that, the drafted policy on palliative care stipulated the need for the coordinator to be responsible for the overall implementation to ensure success (Ministry of Health and Social Services, 2013).

In that regards, few healthcare professionals who are mostly expected to be in close proximity to patients in need of care were trained in palliative care. The training covered areas that are important in the provision of palliative care and include: communication in palliative care; death awareness; breaking the bad news; nutrition and hydration in end of life care; self awareness and burnt out; the dying process and support to family and patient; grieve and bereavement; dealing with common reaction to loss and fear; sexuality and gender issues in palliative care; ethical issues in palliative care; spiritual and cultural issues among others (Ministry of Health and Social Services, 2013),(African Palliative Care Association, 2011), (Chipare, 2015). Similarly, it is the duty of trained palliative care healthcare workers to act as focal people, and providers of psychosocial and spiritual care in a palliative care team. It was also noted by Morrison et al. (2011), that in the absence of palliative care services patients may spend much time in hospitalization and that led to burden of diseases and overspending of public resources.

The proper functioning of palliative care unit should ensure training of healthcare workers in holistic palliative care components which are physical; spiritual, emotional and social needs, basic healthcare, general hygiene and symptom management. However, Davidson et al. (2007) reported a similar situation that, patients with life limiting conditions continue to endure many difficulties related to psychosocial and spiritual needs which affect their health and healing.

Therefore, as a result of declined practice of palliative care service in Namibia, it is anticipated that the patients in need of palliative care may not receive the necessary care in areas of end of life care, particularly care regarding communication in palliative care and breaking the bad news; as demanded by the life limiting illnesses. Similarly, healthcare workers are unaware of the need of palliative care service as there is often no consistent referral process or criteria (African Palliative Care Association, 2009).

As a result of declined palliative care services it is expected that burden of disease in the healthcare sectors, increased morbidity and mortality among patients, may continue to affect patients in life limiting conditions. In the same vein, it is anticipated that workload, burn out and stress among healthcare workers may occur, that result into poor work performance.

There is no available evidence that could describe the rationale for the declined practice of holistic palliative care in the Namibian public health services. It is therefore not known if the declined palliative care service is associated with the competence of healthcare workers on the psychosocial, spiritual aspects of palliative care. Hence, the study aimed to critically analyze the knowledge and practice of healthcare workers, on psychosocial aspects of palliative care service in the public health facilities in Ohangwena region and to identify the training needs.

The aim of the study was to critically analyze the knowledge and practice of healthcare workers on psychosocial aspects of palliative care and identify the training needs.

The findings from the study will inform policy makers, providers and implementers of palliative care services on the current state of psychosocial aspects of palliative care. Furthermore, this study contributed to the main study on the development of the conceptual framework of palliative care services in the Namibian public health services. 
The study was delimited to Ohangwena region where there is high profile of HIV patients and ART defaulter rate. Challenges with patients follow up was reported due to cross border activities with Angola (Ministry of Gender Equality and Child Welfare, 2009).Due to the limited resources such as time and financial constraints, the study was only conducted in Ohangwena region and that limit the generalization of the findings.

Knowledge score could not be computed to measure the degree of competences and to calculate the effect measure (odds ratio) in order to identify causality.

The study included healthcare workers who have been employment for the duration of 30 days and who were allocated at the following departments: Medical/surgical units, children's wards, outpatient/ causality departments, oncology units, tuberculosis units, HIV/AIDS clinics, primary health care outreach program, pharmacies, orthopedics units and social work departments.

The following units were excluded; maternity wards, mental health units, theatre, dental departments, intensive care units (ICU) and departments that were not in direct contact with patients.

\section{Methods}

A cross-sectional analytical study was conducted in the public health facilities in Ohangwena region.

\section{Study population}

The study population was healthcare workers at public healthcare facilities. The target population was healthcare workers that were providing care to patients with life limiting conditions at the eligible units. The population size of healthcare workers was 77 which consisted of medical practioners, nurses, physiotherapists, social workers and pharmacists.

\section{Sampling method}

The sample frames were obtained from the administrators of each respective hospital. A stratified sampling method was used to select participants for each professional category. The sample size was calculated by entering values into the following required criteria in Epi Info StatCalc in order to obtain a desired sample size. The following criteria were used: population size of 77 , expected frequency of $50 \%$, confidence limit of $5 \%$ and confidence level of $95 \%$. A sample size of 65 was obtained however only 56 healthcare workers completed the questionnaires.

\section{Data collection and tools}

A structured questionnaire with closed ended questions containing psychosocial knowledge and practice-based variables was designed for healthcare workers and it was developed in English which is an official language used in the Namibian public health system.

Variables in the measuring tools constituted of socio-demographics status of healthcare workers as well as indicators related to knowledge and practices regarding psychosocial aspects of holistic palliative care.

Validity control measures were assured by consulting experienced researchers at the University of Namibia, and the academic advisors at Texila American University, who offered guidance.

In this study, accurate representation of the total population under study, was identified and scientific sampling methods used to determine reliability as stipulated by Golafshani (2003). The research instrument was validated with experts in the field and through pilot testing in Oshana region, which did not form part of the selected regions. Piloting was done to ensure consistency and to remove duplications as well as spelling mistakes.

\section{Field work}

Questionnaire for healthcare workers was handed over at Ohangwena health region at selected health facilities namely, Eenhana, Okongo and Engela hospital respectively. The selected sites were 
substantiated by regional profiles of most patients who were in need of palliative care and the challenges experienced with anti-retroviral treatment (ART) defaulters.

Self administered questionnaires were handed over by the researcher to participants and were collected after two days. Giving two days was done to ensure that participants understood all questions in order to reduce omissions and also to facilitate response rate. Data was collected during the period of one month after approval to conduct the study.

\section{Data analysis}

Quantitative data was analyzed using SPSS version 24 software. Data was entered into computer to create electronic data base and was cleaned for completeness and accuracy. The descriptive statistics was used to summarize the data. The data was then presented as tables, graphs and charts.

Inferential statistic for categorical variables was done with Pearson Chi-square test to calculate associations. A p-value of less than 0.05 for all analysis was considered statistically significant to reject a null hypothesis.

\section{Ethical issues}

Approval to conduct the study was granted by the research committee of Texila American University. Permission was obtained from the permanent secretary of the Ministry of Health and Social Services (MOHSS) of Namibia, as well as from Ohangwena regional health director. Participants were provided with all necessary information before they decide to sign the consent voluntarily. Participants were advised not to write their names or any form of identity on the form to ensure anonymity. All information was kept safe in a computer which was locked with a password to ensure confidentiality. Participants were informed that they had the right to withdraw at any time with no fear of being victimized.

\section{Results}

A total of 56 participants took part in the study. The mean age of respondents was $36.2 \pm 9.81$. Respondents consisted of 20 (35.7\%) males and 36 (64.3\%) females. Their marital status varied from single 27 (48.2\%), married $26(46.4 \%)$ and widow 3(5.4\%). Among the fifty-six respondents, 2 (3.6\%) were medical officers, 44(77.6\%) nurses, 1 (1.8\%) physiotherapist, 3(5.4\%) social workers and $6(10.7 \%)$ pharmacists.

Table 1. Representing the highest qualification of respondents

\begin{tabular}{|l|l|l|}
\hline Variables: & Frequencies $(\mathrm{n}=56)$ & Percentages (\%) \\
\hline Certificate & 24 & $42.9 \%$ \\
\hline Basic diploma & 9 & $16.1 \%$ \\
\hline Basic degree & 19 & $33.9 \%$ \\
\hline Post graduate diploma & 3 & $5.4 \%$ \\
\hline PhD (doctorate degree) & 1 & $1.8 \%$ \\
\hline
\end{tabular}

From table one above, the highest percentages (42, 9\%) were those with certificates followed by $33.9 \%$ of basic degree holders, while the least (1.8\%) was PhD holders.

The professional rank of the respondents range from pharmacist assistants 4 (7.1\%), senior pharmacist assistants 1 (1.8\%), pharmacist, 1 (1.8\%), social workers 3(5.4\%), principal physiotherapist 1 (1.8\%), medical officers 2 (3.6\%), enrolled nurses 18 (32.1\%), registered nurses $21(37.5 \%)$ and senior registered nurses 5(8.9\%).The respondents mean years of professional experience was $9 \pm 9$.63.The minimum years of professional experiences was 1 year and the maximum was 40 years. Twenty respondents (35.7\%) indicated being involved in leadership while 36 (64.3\%) specified not involved in leadership at the healthcare facility.

Among the respondents, 9 (16.1\%) were studying while 47 (83.9\%) were not studying during the time of data collection. Regarding access to computer, one participant (1.8\%) did not respond, 37 
(66.1\%) had access to computer, while $18(32.1 \%)$ had no access to computer. Two participants (3.6\%) did not respond on the question if they had access to internet, while 39(69.6\%) had access to internet and 15 (26.8\%) did not have access to internet. Two participants (3.6\%) did not respond on the question if they had access to E- library, while 4 (7.1\%) had access to e-library and 50 (89.3\%) did not have access to e-library. Two participants (3.6\%) did not respond on the question if they had access to physical library, while 6(10.7\%) had access to physical library, 48(85.7\%) did not have access to physical library.

With regard to the awareness and practice of palliative care, 38 (67.9\%) indicated being confronted by palliative care situation at work, 18 (32.1\%) reported not confronted by palliative care situation at work. The respondents, 13 (23.2\%) had relative receiving palliative care, 43(76.8\% had no relative who is receiving palliative care. Eight respondents (14.3\%) had focal person for palliative care available at work place while $48(85.7 \%)$ had no focal person for palliative care at work. Out of 56 who participated in the study, 10 (17.9\%) were placed in a palliative care service before, while most 46 (82.1\%) expressed no previous palliative care placement.

From figure one, the highest percentages (47\%) of respondents indicated not allocated to any palliative care team.

\section{Palliative care teams}

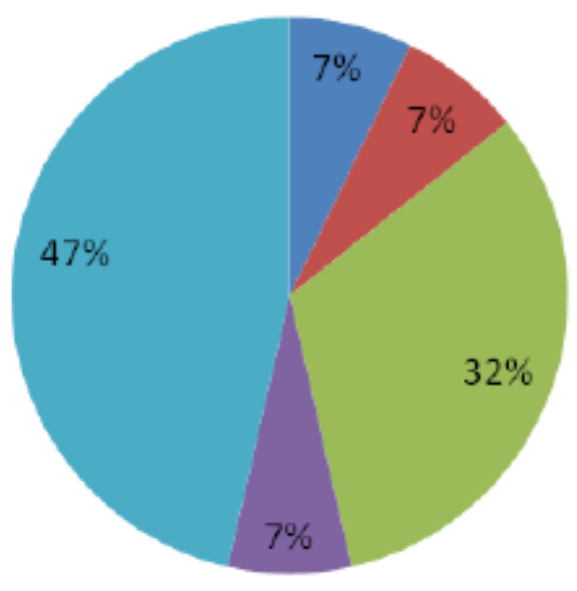

Palliativecare units

Hospital support

Outpatient department or

clinic

Psychosocial supports

Had no palliative care

team

Figure1: Pie chart representing the palliative care team of placement of 56 respondents.

The training of holistic palliative care was attended by 17 (30.4\%) while 39 (69.6\%) specified not trained. Those who responded on the type of training acquired, 2 (3.6\%) had a formal palliative care diploma, 6 (10.7\%) formal degree, 4 (7.1\%) short certificate, 4 (7.1\%) in-service training, while 36 (64.3\%) reported no formal palliative care training. Regarding the duration of palliative care training, 9 (16.1\%) had days of palliative care training, 3 (5.4\%) were trained in weeks, 3 (5.4\%) were trained in months, 5 (8.9\%) were trained in years and $36(64.3 \%)$ had no training. Few 17 (30.4\%) took part in palliative care research compared to most, 39 (69.6\%) who did not take part in research.

With regard to the knowledge and practice of the psychosocial, aspects of palliative care, 17(30.4\%) respondents incorrectly indicated that death and dying should not be discussed with patients while 39(69.6\%) respondents correctly agreed that death and dying should be discussed with patients and family. However, there was a significant association between marital status and if death and dying should be discussed with patients $(\mathrm{p}=0.04)$. Among the 27 single respondents, $5(29.5 \%)$ respondents inappropriately indicated not discussing death and dying while 22 (56.4\%) correctly indicated discussing death and dying as the method of spiritual palliative care. Among the 26 married 
respondents 12 (70.6\%) incorrectly indicated not discussing death with patients while 14 (53.8\%) agreed that death should be discussed with patients.

On the knowledge of practice active listening and nonjudgmental attitude when providing psychosocial palliative care, among the 27 single respondents 26(52\%) indicated correctly to the practice of active listening as a good method of providing psychosocial palliative care, while 1(16.7 $\%$ ) did not think is important to listen to patients without judgment. Among the 26 married respondents 23(46.0\%) indicated that is important to listen to patient without judgment while $3(50.0 \%)$ felt is less important to listen without judgments. Among the 3 widows, $1(2.0 \%)$ indicated that the practice of active listening and nonjudgmental attitude is needed while 2(33.3\%) did not feel that the practice of active listening and nonjudgmental attitude is worth. However, there was a statistical significant association between the knowledge of active listening attitudes and marital status $(\mathrm{P}=0.04)$, with appropriate knowledge among the widows (33.3\%).

With regard to if visitation by spiritual leaders, mentors and pastors should be discouraged, 48(92.3\%) respondents pointed out that visitation by spiritual leaders should not be discouraged, but on the other hand a significant difference existed between discouraging spiritual leaders to visit their clients based on access to E-library $(\mathrm{P}=0.00)$.

On the knowledge of common barriers to breaking the bad news to patients and families in palliative care, there was no significant association identified based on if respondents felt responsible for maintaining the patient's hope ( $>00.05$ ). On the contrary, among the 56 respondents, $42(75.0 \%)$ wrongly indicated that cure is the goal of palliative care, compared to 14 (25.0\%) who indicated that the goal of palliative care is not to cure. In the same way, a significant association between the goal of palliative care and access to internet was specified $(\mathrm{P}=0.04)$ with most $31(73.8 \%)$ of those who had access to internet, incorrectly specified that cure is the goal of palliative care.

The majority 55 (98.2\%) pointed out that the family need to be included in decisions relating to patient care, while $1(1.8 \%)$ felt that the family should not take part in patient care decisions. Thirty three, (58.9\%) responded that photos and personal belongings should not be discouraged when providing palliative care. A significant association between if the use of photos and personal belongings should be discouraged when providing palliative care, was discovered based on marital status ( $\mathrm{P}=0.001)$ with half, (50\%)of the married respondents stated that the use of photos and personal belongings should be discouraged among palliative care patients.

To the question if it is proper to always consider personal own values before the values of patients, the respondents, 16 (28.6\%) incorrectly indicated considering own value compared to 40 (71.4\%) who stated considering the values of patients before their own values. In opposition, a statistical significant association between considering personal own values before the values of patients do exist based on if the respondent participated in palliative care research $(\mathrm{P}=0.02)$.

Regarding the knowledge if it is proper to refer patients to appropriate persons, who can facilitate the practice of specific customs, 50 (89.3\%) of the respondents correctly felt it is proper to refer patients while $6(0.7 \%)$ indicated that it is not proper to refer patients.

In view of, if time and privacy constraints were reasons for not discussing sexuality with patients, out of the 56 respondents, 42 (75.0\%) experienced challenges, while 14 (25.0\%) did not experience challenges. However, respondents' attitudes on sexuality significantly varied based on access to Elibrary, with most $40(80.0 \%)$ of those without access to E-library experiencing challenges ( $p \leq 0.05)$.

On the question to define palliative care for children, 51 (91.1\%) respondents correctly defined palliative care as the active total care of the child's body, mind and spirit, while 5 (8.9\%) did not define palliative care as total care. Conversely, a significant association was established based on being confronted with palliative care at the work place $(\mathrm{P}=0.016)$. On the other hand, $10(17.9 \%)$ respondents correctly indicated that palliative care treatment should continue throughout child treatment, 46 (82.1\%) stated that, palliative care treatment should discontinue. A significant association between if treatment should discontinue was indicated based on palliative care team. 
Respondents who belonged to hospital support team indicated high percentage $75.0 \%$ of discontinuing palliative care when the child receive treatment $(\mathrm{P}=0.031)$.

Among the participants, 45 (80.4\%) indicated that they assess the child's pain according to what the child says while, 18 (32.1\%) indicated that clinical sign is the only method to assess pain in children. A significant associations between assessing child's pain and respondent's research participation was found $(\mathrm{P}=0.036)$.

Out of 56 respondents, 8 (14.3\%) incorrectly categorized codeine phosphate into step one analgesics, while 48 (85.7) correctly placed codeine into steps 2 analgesics. On the contrary, 35 (62.5\%) respondents incorrectly classified morphine, while 21 (37.5\%) correctly classified morphine into step 3 analgesics. Knowledge of morphine classification significantly varied based on the duration of palliative care training $(\mathrm{P}=0.001)$, with $71.4 \%$ of those not trained in palliative care misclassified morphine.

Among the respondents, 47(83.9\%) used a referral form while 9 (16.1\%) stated not using the referral form. However, association between the use of referral form and participants' highest qualification was significant $(\mathrm{P}=0.007)$.

\section{Discussion}

A total of 56 healthcare workers participated in the study that consisted of medical officers, nurses, physiotherapists, social workers and pharmacists. The mean age of healthcare workers was 36.土 9.81). Meanwhile, previous study by Selman, Young, Vermandere, Stirling, \& Leget, (2014), among palliative care physicians, nurses and chaplains, reported a mean age of $48.5 \pm 10.7$, an age older than the age of participants in this study. Younger age as reported in this study could have an implications related to the impact of experience on healthcare worker's knowledge on palliative care. Knowledge implications could influence the quality of psychosocial palliative care provided to patients with life limiting illness. In this study, female participants were more than males while more male participants (64\%) than females were reported for the study on international survey of palliative care researchers and clinicians. The findings are different from the study conducted by Selman et al., (2014). Participant's responses also varied according to their marital status of being either single, married, or widow. The majority (67.9\%) reported being confronted with palliative care situation in the work place even though most (69.6\%) specified not trained in palliative care. The fact that they encounter palliative care situations without proper training, may affect the quality of holistic palliative care provision. Similarly, Selman et al. (2014) also noted that, cancer patients' spiritual needs were not assessed well by the healthcare team as a result of inadequate knowledge and skills on psychosocial palliative care among the healthcare workers. The respondents mean years of professional experience was 9 years which is a sufficient period to gain skills. The findings is congruent with the study by Bronheim, Can, \& Anthony (2015), who stipulated that healthcare worker's experience influenced their commitment to provide quality services and supports of patients.

Although the majority of respondents indicated being confronted by palliative care situations, only few had their relatives that received palliative care. This means, healthcare workers experienced palliative care situations more at work than at home. Lack of the focal person for palliative care in the healthcare facility as sighted by most respondents, may result into poor supervision and support which will eventually affect the provision of psychosocial palliative care to the patients. In the same vein, it was stipulated by Andershed et al. (2007) that, palliative care activities require more managerial and leadership training and support for palliative healthcare workers.

In this study, it was noted that the placement of healthcare workers in palliative care team was not supportive of psychosocial palliative needs of patients. The absence of palliative care placement could prevent healthcare workers from gaining experiences and skills to assist patients in need of palliative care and that could also affect the quality of care. It was also stipulated in (Global Partners in Care, 2014) that, clinical placements were regarded as appropriate for both healthcare workers at the time of 
training, in order to gain experience with palliative care practice. The gained experience could be adapted in their own practices in future to ensure proper continuity of patients care.

The training of holistic palliative care was attended by few in relation to the majority. Those who responded on the type of training acquired, few had formal palliative care training. Training increase the ability of the practioners to build resilience to stress and burnt out. Similarly and as emphasized by Back, Steinhauser, Kamal, \& Jackson (2016), lack of training can expose healthcare professionals to burnout and psychological exhaustion that will hamper their ability to support patients. The majority did not take part in palliative care research and that may lead to the failure to account for expenditures and outcome in the absence of monitoring and evaluation of their activities. A study by Morrison et al.(2011), emphasized the need for palliative care studies for economic and policy formulation purposes.

Regarding if death and dying should be discussed with patients and family during palliative care, single and married persons equally indicated discussing death and dying as the method of spiritual palliative care with patients, which is the correct method. There was a significant association between marital status and knowledge of discussing death $(\mathrm{p}=0.04)$. Similarly, there was a statistical significant association between active listening attitudes of participants and marital status. $(\mathrm{P}=0.04)$. Participants had no equal knowledge of spiritual care with respect to their marital status.

Meanwhile, a significant difference of knowledge also existed between discouraging spiritual leaders to visit their clients based on access to E-library and to physical library $(\mathrm{P}=0.00)$ and $(\mathrm{P}=0.030)$ respectively. This means that participants had no equal chance of knowledge on this matter based on library access.

Lack of proper information on spiritual palliative care could affect the way a service is rendered to patients facing end of life. Another study on the experience of patients about the fear of death indicated that lack of information on their prognosis led to fear of dying. It is therefore imperative that healthcare workers discuss death with patients and family to prepare them for end of life expectations (Gardiner et al., 2009).

Healthcare professionals need to be mindful when breaking bad news to patients who are faced with challenges of illness prognosis in order to maintain hope. Moreover, it was suggested by Olsman, Leget, Onwuteaka-Philipsen, \& Willems (2014), that healthcare professionals should be realistic by combining hope with the truth, in order to help patients to cope with hopeless situations.

Conversely, there was a significant association between the knowledge on the goal of palliative care and access to internet $(\mathrm{P}=0.04)$. Most of those who had access to internet incorrectly specified that cure is the goal of palliative care

It is crucial that the goal of palliative care be known equally among healthcare workers and to be mindful when accessing information from the internet in order to avoid misconceptions.

The majority of the respondents knew that the family need to be included in decisions related to patient care. On the contrary, half of the married respondents stated that photos and personal belongings should be discouraged among palliative care patients, which is a bad practice.

Allowing patients and family to use memories of their loved ones may enhance hope that could be easily destroyed by disclosure of the truth. Another study by Olsman et al. (2014) noted that disclosure of medical information or use of the word 'palliative care' wipe out hope.

On the knowledge, if it is proper to always consider personal own values before the values of patients. Few respondents, incorrectly indicated considering own value. Respondent's knowledge differs across holistic palliative care training and research participation. The response of the respondents could be worrisome if they consider their own values in expense of patient values. It was suggested by Tan, Seah, Chua, Lim, \& Phua (2014), that patients need to establish their own values even if their wishes differ with their family members on treatment expectations.

The practice of referring patients to appropriate person who can facilitate the practice of specific norms was correctly indicated by the majority. However, there was a statistically significant 
association based on access to computer. It is essential that healthcare workers should respect the diversity of culture and work in harmony with patients and family in order to facilitate wellbeing.

Most of the respondents stated that, time and privacy constraints prevent them from discussing sexuality with patients. Those with limited access to E-library felt limited to discuss sexuality with patients. However sexuality and gender issues were observed as part of palliative care training for healthcare workers (African Palliative Care Association, 2012). Failure to discuss sexuality issues may expose patients to anxiety and that may affect their recovery.

When asked to define palliative care for children, majority correctly defined palliative care as the active total care of the child's body, mind and spirit.

On the contrary, the majority of respondents who belonged to hospital support team incorrectly indicated that palliative care treatment should discontinue when the child receive treatment on life limiting illness. Hospital support team could not define palliative care for children.

In the absence of proper definition for pediatric palliative care, holistic palliative care will not be possible from the time of diagnose throughout treatment and that may affect the prognosis of a child suffering from life limiting illness. A study by Morgan (2009), noted that pediatric palliative care should begin at the time of diagnosis if death is clearly expected, so that the child and parents are prepared for the outcome of illness.

Most participants indicated the correct practice of assessing the child's pain according to what child says, however, a significant associations of their practice was identified based on respondents 's research participation. This means that, the distribution of knowledge regarding assessing pain in children was not equal among the respondents. Another study by Bronheim et al.(2015), also noticed that lack of palliative care service for children affects children’s recovery.

Knowledge on how to classify morphine significantly varied based on the duration of palliative care training $(\mathrm{P}=0.001)$, with most of those indicated not trained in palliative care misclassified morphine. However it was suggested in Malawi, that a hospital must have full-time, designated palliative care services and trained staff; registered palliative care patients; a regular supply of drugs, including morphine (Global Partners in Care, 2014).

With regard to the referral system, the majority indicated used a referral form, however, association between the use of referral form and participants' highest qualification was significant $(\mathrm{P}=0.007)$. It is therefore imperative if healthcare workers had equal exposure to the coordinated network system. The presence of proper referral network with functioning referral tools such as referral forms will enhance continuous follow up. It was also stated in The United Republic of Tanzania (2005), that the referral form should contain all the relevant information, including diagnosis and instructions on the current management and treatment in order to provide continuum of care at various levels.

\section{Conclusion}

This study concludes that there is inadequate knowledge and practice regarding psychosocial aspects of palliative care among healthcare workers in Ohangwena region. Lack of knowledge was based on lack of palliative care training among healthcare workers. Secondly, inadequate placement in palliative care team as well as limited exposure to internet also contributed to unequal knowledge distribution among healthcare workers.

\section{Recommendations}

This study recommends the following aspects as indicated by the findings in the study:

Organize workshops and seminars to train healthcare workers on the essential aspects of holistic palliative care especially on spiritual care, sexuality and gender issues, as well as cultural issues in palliative care.

Develop a standardized curriculum to educate healthcare workers about children's pain assessment and management as well as the use of opioids drugs, especially morphine.

Palliative care training should consider difference in age, as well as marital status to cater for variability. 
Facilitate the provision of internet and E- library to facilitate knowledge distribution of psychosocial aspects of palliative care among various healthcare professionals.

Appoint focal persons at district level to coordinate palliative care teams especially for the hospital palliative care services.

Establish a proper referral network with functioning referral tools such as referral forms, to ensure continuity of care.

\section{Acknowledgements}

The authors declare that no funds received for this study and no conflict of interest.

\section{References}

[1]. African Palliative Care Association. (2009). Palliative care situational analysis report. Ministry of Health and Social Services. Windhoek.

[2]. African Palliative Care Association. (2011). African palliative care association standards for providing quality palliative care across Africa. Retrieved from www.africanpalliativecare.org

[3]. African Palliative Care Association. (2012). Palliative care core curriculum. Uganda. Retrieved from www.africanpalliativecare.org

[4]. African Palliative Care Association. (2013). Palliative care for women living with HIV and cervical cancer. Kampala, Uganda. Retrieved from www.africanpalliativecare.org

[5]. Andershed, B., Sims, M. T., Authors, F., Davison, G., Davidson, J. E., Powers, K., ... Fish, A. (2007). Palliative care teams and organisational capability. Palliative Medicine, 24(1), 60-67. http://doi.org/10.1108/13527590210433357.

[6]. APC. (2014). Effective methods of teaching palliative care. A guide for educators and trainers in Africa. Kampala, Uganda. Retrieved from www.africanpalliativecare.org

[7]. Back, A. L., Steinhauser, K. E., Kamal, A. H., \& Jackson, V. A. (2016). Building resilience for palliative care clinicians $\square$ : An approach to burnout prevention based on individual skills and workplace factors. Journal of Pain and Symptom Management. http://doi.org/10.1016/j.jpainsymman.2016.02.002.

[8]. Bronheim, S. M., Can, E., \& Anthony, B. J. (2015). Improving care coordination for African American and Hispanic children with special healthcare needs. Journal of Children's Services, 10(1), 45-56. http://doi.org/10.1108/JCS-04-2014-0023.

[9]. Chipare, M. (2015). A health education programme to enhance knowledge and communication skills of health care workers serving people living with HIV/AIDS on HAART in Namibia. University of Namibia. Retrieved from www.unam.na

[10]. Davidson, J. E., Powers, K., Hedayat, K. M., Tieszen, M., Kon, A. a, Shepard, E., ... Armstrong, D. (2007). Clinical practice guidelines for support of the family in the patient-centered intensive care unit: American College of Critical Care Medicine Task Force 2004-2005. Critical Care Medicine, 35(2), 605-622. http://doi.org/10.1097/01.CCM.0000254067.14607.EB.

[11]. Gardiner, C., Gott, M., Small, N., Payne, S., Seamark, D., Barnes, S., ... Ruse, C. (2009). Living with advanced chronic obstructive pulmonary disease: patients concerns regarding death and dying. Palliative Medicine, 23(8), 691-697. http://doi.org/10.1177/0269216309107003.

[12]. Global Partners in Care. (2014). For clinical placement in hospice and palliative care in Africa. Alexandria. Retrieved from www.globalpartnersincare.org

[13]. Golafshani, N. (2003). Understanding reliability and validity in qualitative research. The Qualitative Report, 8(4), 597-607. http://doi.org/10.3367/UFNr.0180.201012c.1305.

[14]. HPV Information Centre. (2014). Human papillomavirus and related cancers, Fact Sheet. Retrieved from www.hpvcentre.net

[15]. Ministry of Gender Equality and Child Welfare. (2009). Strengthening commitment and leadership of government to expand HIV and AIDS response, gender issues and women's empowerment. Journal of Petroleum Science and Engineering, 67(3-4), 179. http://doi.org/10.1016/S0920-4105(09)00154-5.

[16]. Ministry of Health and Social Services. (2013). Palliative care training report. Directorate of Ohangwena region. Windhoek. 
[17]. Morgan, D. (2009). Caring for dying children: Assessing the needs of the pediatric palliative care nurse. Pediatric Nursing, 35(2), 86-90.

[18]. Morrison, R. S., Dietrich, J., Ladwig, S., Quill, T., Sacco, J., Tangeman, J., \& Meier, D. E. (2011). The care span: Palliative care consultation teams cut hospital costs for medicaid beneficiaries. Health Affairs, 30(3), 454-463. http://doi.org/10.1377/hlthaff.2010.0929.

[19]. Olsman, E., Leget, C., Onwuteaka-Philipsen, B., \& Willems, D. (2014). Should palliative care patients’ hope be truthful, helpful or valuable? An interpretative synthesis of literature describing healthcare professionals' perspectives on hope of palliative care patients. Palliative Medicine, 28(1), 59-70. http://doi.org/10.1177/0269216313482172.

[20]. Selman, L., Young, T., Vermandere, M., Stirling, I., \& Leget, C. (2014). Research priorities in spiritual care: An international survey of palliative care researchers and clinicians. Journal of Pain and Symptom Management, 48(4), 518-531. http://doi.org/10.1016/j.jpainsymman.2013.10.020.

[21]. Tan, A., Seah, A., Chua, G., Lim, T. K., \& Phua, J. (2014). Impact of a palliative care initiative on endof-life care in the general wards: a before-and-after study. Palliative Medicine, 28(1), $34-41$. http://doi.org/10.1177/0269216313484379.

[22]. The United Republic of Tanzania. (2005). Guidelines for home based care services. Ministry of Health. Dar es Salaam. 